\title{
A sunny outlook
}

\author{
Using advanced technologies to improve photoinduced charge transfer and light harvesting is essential \\ in the development of high-efficiency solar cells. Once the problem of energy storage has been \\ overcome, solar energy is sure to become the world's major source of sustainable energy.
}

The field of photovoltaics has made steady theoretical and technological progress since the discovery of photoinduced current by French physicist Alexandre Becquerel in $1839^{1}$. Global warming and the impending exhaustion of fossil fuels are placing increasing demand on sustainable energy, in particular energy harvested from sunlight. Scientists have investigated a wide variety of photovoltaic cells in an attempt to increase power-conversion efficiencies and reduce fabrication costs. This month, Nature Photonics presents a Focus Issue on photovoltaics, featuring a collection of two Review Articles, one Progress Article, two Commentaries and an Interview.

Organic photovoltaic cells - particularly those based on polymers - have attracted considerable attention in recent years, owing to their low cost and potentially high power-conversion efficiencies. On page 153, Yang Yang and co-workers explain that the highest-efficiency polymer solar cells are those based on polymer-fullerene systems, whose power-conversion efficiencies are approaching 10\%. Fullerene derivatives have become the standard n-type molecules for use in polymer solar cells owing to their strong electronegativity and high electron mobility.

Coincidentally, on page 180 of this issue, Yang and co-workers report a tandem polymer solar cell based on a low-bandgap conjugated polymer with a record powerconversion efficiency of $8.62 \%$, which gives significant cause for optimism in this field.

Another interesting development in the field of photovoltaics is the dye-sensitized solar cell, in which the functions of electron transport, light absorption and hole transport are each handled by separate materials. The dye-sensitized solar cell is the only photovoltaic cell to take advantage of redox reactions. Using this approach, scientists have been able to increase powerconversion efficiencies to around $11 \%{ }^{2}$. On page 162, Michael McGehee and coworkers describe strategies for improving the power-conversion efficiency of dye-sensitized solar cells. They list several promising redox systems, some of which could lead to power-conversion efficiencies of more than $15 \%$.

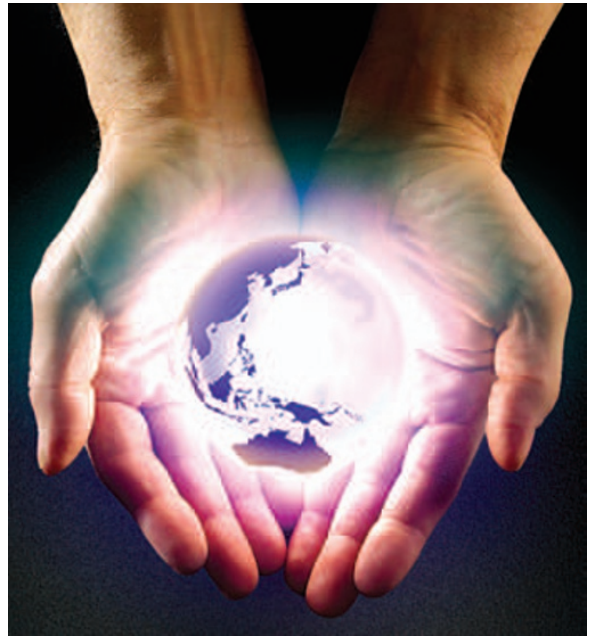

Another way to achieve efficient light harvesting is by appropriately engineering the cell. On page 133, Edward Sargent summarizes the recent progress in the development of solar cells based on colloidal quantum dots, whose diameter can be tuned to offer full-spectrum solar harvesting. Sargent overviews various optoelectronic device architectures, including Schottky, depleted heterojunction and tandem cells, and also describes the quantum funnel cell ${ }^{4}$, a device recently proposed to augment the electronextracting driving force by generating a built-in gradient in the conduction band through a quantum-tuned increase in bandgap towards the back of the cell.

One particular highlight of this Focus

The intermediate-band solar cell is an emerging photovoltaic technology that was first demonstrated in $2004^{3}$ and consists of an intermediate-band material sandwiched between two ordinary n- and p-type semiconductors. Incident photons with energies lower than the gap between the conduction and valence bands can be absorbed by a two-step transition: first between the valence band and the intermediate band, then between the intermediate band and the conduction band. On page 146, Antonio Luque et al. summarize the research history and future challenges in this field. They anticipate that bulk intermediate-band solar cells might exceed the power-conversion efficiencies of current thin-film photovoltaic cells.

Light harvesting can be improved by using a thicker layer of material to increase the optical pathlength, but this is in conflict with current efforts to reduce solar module costs by decreasing the thickness of the cell. On page 130, Martin Green and Supriya Pillai describe how surface plasmon polaritons could be the answer to this trade-off, as their propagation direction is along the metaldielectric interface and therefore not dependent on the cell thickness. They also point out that appropriate shaping and positioning of metallic nanoparticles could efficiently enhance performance in photovoltaic cells.
Issue is the interview on page 136 with Stuart Wenham, chief technology officer at Suntech, one of the world's largest producers of silicon solar panels. He reveals that Suntech has recently achieved a power-conversion efficiency of $20.3 \%$ in its current commercial silicon solar cells. The primary goal for solar cell manufacturers is cost reduction, and Wenham points out that this can be achieved both by improving power-conversion efficiencies and by reducing fabrication costs. Wenham shares with us the steps taken by Suntech to reduce the cost of its solar cells, as well its outlook on the photovoltaic industry as a whole. The photovoltaic market will truly expand when the cost of solar-harvested energy falls below that of fossil-fuelgenerated energy.

By the end of this century, the world's energy requirements will be four times what they are at present. Although rapid progress in photovoltaic research has already provided substantial positive growth, the main challenge now faced by the industry is to develop techniques for storing the energy harvested during the day for use at night. Only then will solar energy rise to take its place a major provider of sustainable energy.

References

1. Becquerel, E. Comptes Rendus 9, 561-567 (1839).

2. Chiba, Y. et al. Jpn. J. Appl. Phys. 45, 638-640 (2006)

3. Luque, A. et al. J. Appl. Phys. 96, 903-909 (2004).

4. Kramer, I. J., Levina, L., Debnath, R., Zhitomirsky, D. \&

Sargent, E. H. Nano Lett. 11, 3701-3706 (2011). 